\title{
Assessment of Doripenem, Meropenem, and Imipenem against Respiratory Isolates of Pseudomonas aeroginosa in a Tertiary Care Hospital of North India
}

\author{
Arti Negi, Mridu Anand, Avinash Singh, Awadhesh Kumar, Chinmoy Sahu, Kashi Nath Prasad \\ Department of Microbiology, Sanjay Gandhi Postgraduate Institute of Medical Sciences, Lucknow, Uttar Pradesh, India
}

\section{Abstract}

Objective: Pseudomonas aeruginosa is one of the leading pathogen causing healthcare-associated infections, particularly in immunocompromised and critically ill patients. The development of carbapenem resistance in P. aeruginosa infections is worrisome. Data specifically comparing the susceptibility of the three available carbapenems are lacking in the Indian subcontinent. Materials and Methods: We evaluated the minimum inhibitory concentrations (MICs) of the three commonly used carbapenems-imipenem, meropenem, and doripenem against, 435 P. aeruginosa isolates obtained from respiratory samples and compared their susceptibility patterns to determine the best possible carbapenem among those available that may be used in combination regimes. Results: Overall, $222(51.0 \%)$ of isolates were susceptible to doripenem followed by imipenem 206 (47.3\%) and meropenem 195 (44.8\%), respectively. Two hundred and sixty-two (60.23\%) strains were intermediate or resistant to at least one carbapenem. The $\mathrm{MIC}_{90}$ of all three carbapenems was $>32 \mu \mathrm{g} / \mathrm{ml}$ while the $\mathrm{MIC}_{50}$ of meropenem was $16 \mu \mathrm{g} / \mathrm{ml}$ which was higher than $\mathrm{MIC}_{50}$ of both imipenem $(4 \mu \mathrm{g} / \mathrm{ml})$ and doripenem $(2 \mu \mathrm{g} / \mathrm{ml})$. Conclusion: Our study revealed that doripenem exerted better in vitro activity against the tested bacteria compared to imipenem and meropenem, but the difference was not statistically significant.

Keywords: Doripenem, imipenem, meropenem, minimum inhibitory concentration, Pseudomonas aeruginosa

\section{INTRODUCTION}

Pseudomonas aeruginosa is one of the leading pathogens causing healthcare-associated infections particularly in immunocompromised and the critically ill patients. It is one of the most prevalent pathogens in nosocomial pneumonia, especially in patients with high-risk factors such as mechanical ventilation or catheter intubation. It has been acquiring multidrug resistance (MDR) at an alarming rate, raising much clinical concern as effective antimicrobial agents are limited and dwindling. ${ }^{[1]}$ The development of carbapenem resistance, against $P$. aeruginosa infections is worrisome. ${ }^{[2]}$

Carbapenem resistance can be due to the production of enzymes, such as AmpC or a metallo- $\beta$-lactamase; overexpression of efflux pumps; porin deficiencies; or target site alterations. One of the major risk factors for carbapenem resistance is carbapenem use itself resulting in selective pressure on bacterial populations. ${ }^{[3]}$

Literature suggests, doripenem is an antipseudomonal carbapenem that has greater in vitro activity against

\begin{tabular}{|l|l|}
\hline \multicolumn{3}{c|}{ Access this article online } \\
\hline Quick Response Code: & Website: \\
& www.ijccm.org \\
& \\
\end{tabular}

P. aeruginosa isolates than other carbapenems and is less likely to select for carbapenem-resistant strains under experimental conditions. ${ }^{[4,5]}$ Its $1-\beta$-methyl side chain provides resistance to dehydropeptidase so that this molecule does not require the addition of cilastatin for protection from this enzyme. It is also remarkably stable after reconstitution, increasing the opportunity for prolonged infusion. The recommended dosing for doripenem is $0.5 \mathrm{~g}$ every $8 \mathrm{~h}$ (administered through 1 or $4 \mathrm{~h}$ infusions). ${ }^{[5]}$

Doripenem has been described as having the favorable attributes of both imipenem and meropenem against both Gram-positive and Gram-negative bacteria. ${ }^{[6]}$ Data

Address for correspondence: Dr. Chinmoy Sahu, Department of Microbiology, Sanjay Gandhi Postgraduate Institute of Medical Sciences, Lucknow, Uttar Pradesh, India. E-mail: sahu.chinmoy@gmail.com

This is an open access article distributed under the terms of the Creative Commons Attribution-NonCommercial-ShareAlike 3.0 License, which allows others to remix, tweak, and build upon the work non-commercially, as long as the author is credited and the new creations are licensed under the identical terms.

For reprints contact: reprints@medknow.com

How to cite this article: Negi A, Anand M, Singh A, Kumar A, Sahu C, Prasad KN. Assessment of doripenem, meropenem, and imipenem against respiratory isolates of Pseudomonas aeroginosa in a tertiary care hospital of north India. Indian J Crit Care Med 2017;21:703-6. 
specifically comparing the susceptibility of the three available carbapenems are lacking. Therefore, we evaluated the minimum inhibitory concentrations (MICs) of the three commonly used carbapenems; imipenem, meropenem, and doripenem against isolates of $P$. aeruginosa obtained from respiratory samples and compared their susceptibility patterns.

\section{Materials and Methods}

This prospective study was conducted in the Department of Microbiology at a tertiary care referral hospital of North India from August 2015 to September 2016. We studied in vitro susceptibility of $435 P$. aeruginosa isolates recovered from respiratory samples against carbapenems; imipenem, meropenem, and doripenem. These samples included sputum, endotracheal aspirate (ETA), bronchoalveolar lavage (BAL), and mini BAL from patients with suspected respiratory tract infections. The samples were processed semiquantitatively, and colony counts of $10^{5} \mathrm{CFU} / \mathrm{ml}$ were taken as pathogenic. Samples that yielded $P$ aeruginos $a$ identified using standard techniques ${ }^{[7]}$ and confirmed by an automated identification system (Phoenix ${ }^{\mathrm{TM}}$ 100, BD Biosciences, Maryland, USA) were included for further study.

Antimicrobial susceptibility testing and determination of MIC for the 3 carbapenems was done on Mueller Hinton agar using E-test strips (AB Bio Merieux, France). The interpretation was done according to Clinical and Laboratory Standards Institute (CLSI) guidelines ${ }^{[8]}$ and results were compared with European Committee on Antimicrobial Susceptibility Testing (EUCAST) guidelines ${ }^{\left[{ }^{[9]}\right.}$ P. aeruginosa strains were classified as MDR, extensively drug-resistant (XDR), and pandrug resistant (PDR), according to the classification proposed by Magiorakos et al. ${ }^{[10]}$

\section{RESULTS}

A total of $435 P$. aeruginosa were isolated from respiratory samples comprising 216 (49.66\%) sputum, 208 (47.82\%) ETA, $10(2.30 \%)$ BAL, and $1(0.23 \%)$ mini BAL. One hundred and forty-one (32.41\%) of the samples were polymicrobial. $15.40 \%$ (67) of the isolates were MDR, $42.99 \%$ (187) were XDR, and $1.61 \%$ (7) were PDR while the remaining 40\% (174) isolates were sensitive to carbapenems. Overall $222(51.0 \%)$ of isolates were susceptible to doripenem followed by imipenem 206 (47.3\%) and meropenem 195 (44.8\%), respectively. Two hundred and sixty-two $(60.23 \%)$ strains were intermediate or resistant to at least one carbapenem.

Carbapenem MIC distributions $\left(\mathrm{MIC}_{50}\right.$ and $\mathrm{MIC}_{90}$ ) for these strains are given in Table 1. Doripenem MICs were lower than those of imipenem or meropenem. The $\mathrm{MIC}_{90}$ of all three carbapenems was $>32 \mu \mathrm{g} / \mathrm{ml}$ while the $\mathrm{MIC}_{50}$ of meropenem was $16 \mu \mathrm{g} / \mathrm{ml}$ which was higher than $\mathrm{MIC}_{50}$ of both imipenem $(4 \mu \mathrm{g} / \mathrm{ml})$ and doripenem $(2 \mu \mathrm{g} / \mathrm{ml}) . \mathrm{MIC}_{50}$ of meropenem, imipenem, and doripenem was significantly different $(P=0.002)$. A diagrammatic representation of the cumulative MIC values of imipenem, meropenem, and doripenem has been depicted in Figure 1. We also evaluated doripenem sensitivity in imipenem and meropenem nonsusceptible isolates [Table 2]. Among the doripenem-resistant isolates $(n=204), 10(4.9 \%)$ were sensitive to imipenem, $1(0.4 \%)$ was sensitive to meropenem, and $8(3.9 \%)$ were sensitive to both imipenem and meropenem. With respect to the meropenem-resistant isolates $(n=233)$, $11(4.7 \%)$ were sensitive to imipenem, $20(8.6 \%)$ were sensitive to doripenem, and $8(3.4 \%)$ were sensitive to both imipenem and doripenem. Of the 216 imipenem-resistant isolates, $3(1.3 \%)$ were sensitive to meropenem, $22(10.1 \%)$ were sensitive to doripenem, and $6(2.7 \%)$ were sensitive to both meropenem and doripenem as shown in Table 2. On comparing susceptibility results of imipenem, meropenem, and doripenem using CLSI and EUCAST breakpoints, there was no significant change in the sensitivity rate of meropenem $(195 ; 44.8 \%$ by CLSI vs. $194 ; 44.6 \%$ by EUCAST). However, in case of imipenem, the sensitivity by CLSI was 206 (47.3\%) while that by EUCAST was 218 (50.1\%). Similarly, $222(51.0 \%)$ isolates were sensitive to doripenem by CLSI while 205 (47.5\%) were sensitive by EUCAST. This difference of sensitivities in case of imipenem and doripenem was not statistically significant $(P=0.60,0.90,0.37$ for imipenem, meropenem and doripenem, respectively). There were $3(0.69 \%)$ isolates in case of imipenem, $15(3.5 \%)$ in case of meropenem and $14(3.2 \%)$ in case of doripenem which fell within the gap between the defined range of sensitive and resistant and hence remained unclassified, as there is no intermediate category defined in EUCAST.

\section{Discussion}

Treatment options for resistant $P$. aeruginosa infections are restricted, and combination therapy with other antimicrobial

\begin{tabular}{|c|c|c|c|}
\hline Antibiotic & Range & $\mathrm{MIC}_{50}$ & $\mathrm{MIC}_{90}$ \\
\hline Meropenem & $0.019->32$ & 16 & $>32$ \\
\hline Imipenem & $0.04->32$ & 4 & $>32$ \\
\hline Doripenem & $0.012->32$ & 2 & $>32$ \\
\hline
\end{tabular}

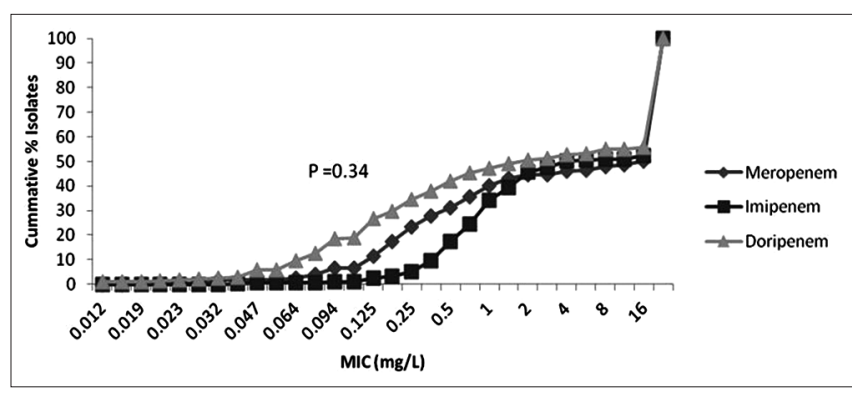

Figure 1: Distribution of cumulative percentage isolates and minimum inhibitory concentrations of doripenem, meropenem, and imipenem among Pseudomonas aeruginosa isolates 
Negi, et al:: Assessment of carbapenems against Pseudomonas aeroginosa

\begin{tabular}{|c|c|c|c|c|c|c|}
\hline Antibiotic resistance & $\begin{array}{c}\text { Imipenem } \\
\text { sensitive (\%) }\end{array}$ & $\begin{array}{l}\text { Meropenem } \\
\text { sensitive (\%) }\end{array}$ & $\begin{array}{c}\text { Doripenem } \\
\text { sensitive (\%) }\end{array}$ & $\begin{array}{c}\text { Imipenem + } \\
\text { doripenem } \\
\text { sensitive (\%) }\end{array}$ & $\begin{array}{l}\text { Meropenem } \\
+ \text { imipenem } \\
\text { sensitive (\%) }\end{array}$ & $\begin{array}{c}\text { Doripenem + } \\
\text { meropenem } \\
\text { sensitive (\%) }\end{array}$ \\
\hline Doripenem resistant $(n=204)$ & $10(4.9)$ & $1(0.4)$ & - & - & $8(3.9)$ & - \\
\hline Meropenem resistant $(n=233)$ & $11(4.7)$ & - & $20(8.6)$ & $8(3.4)$ & - & - \\
\hline Imipenem resistant $(n=216)$ & - & $3(1.3)$ & $22(10.1)$ & - & - & $6(2.7)$ \\
\hline
\end{tabular}

Meropenem vs Doripenem $(P$ value $=0.0754)$; Imipenem verses Doripenem $(P$ value $=0.37)$

agents has been suggested time and again as a potential management strategy. In particular, synergism between colistin/carbapenem has been demonstrated in several studies despite the apparent resistance of $P$. aeruginosa to carbapenems. ${ }^{[1]}$ However, the comparative data regarding the susceptibility of $P$. aeruginosa to the available carbapenems is lacking worldwide. Hence, this study was undertaken as it is imperative to determine the best possible carbapenem among those available that may be used in combination regimes.

In this study, $60.23 \%$ (262) of $P$. aeruginosa strains responsible for respiratory infections at our hospital were intermediate-susceptible or resistant to at least one of the carbapenems; imipenem, meropenem, or doripenem. This is much higher than the isolates studied by Luyt et al. ${ }^{[4]}$ who found $40 \%$ of their isolates were resistant.

We also found that in our isolates MICs of doripenem were lower than imipenem or meropenem MICs [Table 1 and Figure 1] although the difference was not statistically significant in case of $\mathrm{MIC}_{90}, \mathrm{MIC}_{50}$ of meropenem, imipenem, and doripenem showed a significant difference $(P=0.002)$. This is similar to several other studies..$^{[4,11,12]}$ In a study by Luyt et al., ${ }^{[4]}$ similar results were found with respect to the MICs, but their difference was statistically significant both in case of $\mathrm{MIC}_{90}$ and $\mathrm{MIC}_{50}$, implying that doripenem was clearly superior to the other two carbapenems with regard to $P$ aeruginosa. $\mathrm{Hu}$ et al. ${ }^{[1]}$ studied only multidrug-resistant $P$ aeruginosa isolates with a high percentage of carbapenem resistance and found that doripenem still performed better than the other two carbapenems in vitro. In an Indian study by Goyal et al.$^{[13]}$ also, doripenem had an 84.2-fold lower MIC towards $P$. aeruginosa isolates $(0.38 \mathrm{mg} / \mathrm{L})$ than meropenem $(>32 \mathrm{mg} / \mathrm{L})$. One reason why doripenem is more potent than meropenem and imipenem might be due to its higher affinity for penicillin-binding protein (PBP2) and PBP3 in P. aeruginosa. ${ }^{[14]}$ In contrast, in a study conducted by Bretonnière et al. ${ }^{[11]} \mathrm{MIC}$ values of meropenems were lower than to the other two carbapenems.

In our study, the MIC range $(\mathrm{mg} / \mathrm{L})$ was; $0.012->32$ for doripenem, 0.04->32 for imipenem and 0.019->32 for meropenem. This is similar to several other studies. ${ }^{[15-17]}$ In the present study $\mathrm{MIC}_{50}$ and $\mathrm{MIC}_{90}$ data also revealed doripenem to be the most active carbapenem tested against P. aeruginosa clinical isolates. However, $\mathrm{MIC}_{50}$ and $\mathrm{MIC}_{90}$ of doripenem ( 2 and $>32 \mathrm{mg} / \mathrm{L}$, respectively) were higher than those reported from previous studies. ${ }^{[4,13,16]}$ Similar findings were seen in $\mathrm{MIC}_{50}$ and $\mathrm{MIC}_{90}$ of imipenem and meropenem.
This may be due to the antibiotic selection pressure promoted by inappropriate dosage and duration of the carbapenems.

In our study, $8.6 \%$ of $P$. aeruginosa isolates tested as nonsusceptible to meropenem were susceptible to doripenem and $10.1 \%$ imipenem resistant isolates were sensitive to doripenem. This is interestingly higher than the pioneer study conducted by Pillar et al. ${ }^{[17]}$ In their study, $13 \%$ of $P$. aeruginosa isolates tested as nonsusceptible to imipenem, were susceptible to doripenem. This may be due to OprD mutations, often involved in resistance to imipenem but not for meropenem or doripenem which is usually due to increased efflux or production of $\beta$ lactamases. However, the mechanisms of resistance in Pseudomonas are complex, and several mechanisms may be involved simultaneously. ${ }^{[1]}$ When we compared susceptibility results of meropenem and imipenem with doripenem using CLSI, EUCAST breakpoints, no significant variations in sensitivities were seen. This is in concordance with several other studies. ${ }^{[14,18]}$

\section{ConcLusion}

The high rate of drug resistance in $P$. aeruginosa is alarming, and it is crucial to screen for carbapenem resistance prior initiation of antibiotic therapy. Our study revealed that although doripenem exerted better in vitro activity against the tested bacteria compared to imipenem and meropenem, the difference was not statistically significant. However, in few isolates, as demonstrated in our study, susceptibility test to one carbapenem could not predict susceptibility to the other drugs in this class, and hence, the MIC for each carbapenem should be determined separately in resistant/intermediate MIC when facing a potentially difficult to treat infection especially in resourceful settings. MIC values can help in guiding the clinicians to use combination therapy or higher/more frequent recommended dosing of carbapenems.

\section{Financial support and sponsorship}

Nil.

\section{Conflicts of interest}

There are no conflicts of interest.

\section{References}

1. Hu YF, Liu CP, Wang NY, Shih SC. In vitro antibacterial activity of rifampicin in combination with imipenem, meropenem and doripenem against multidrug-resistant clinical isolates of Pseudomonas aeruginosa. BMC Infect Dis 2016;16:444. 
2. Castanheira M, Deshpande LM, Costello A, Davies TA, Jones RN. Epidemiology and carbapenem resistance mechanisms of carbapenem-non-susceptible Pseudomonas aeruginosa collected during 2009-11 in 14 European and Mediterranean countries. J Antimicrob Chemother 2014;69:1804-14.

3. Wolter DJ, Lister PD. Mechanisms of ß-lactam resistance among Pseudomonas aeruginosa. Curr Pharm Des 2013;19:209-22.

4. Luyt CE, Aubry A, Lu Q, Micaelo M, Bréchot N, Brossier F, et al. Imipenem, meropenem, or doripenem to treat patients with Pseudomonas aeruginosa ventilator-associated pneumonia. Antimicrob Agents Chemother 2014;58:1372-80.

5. Bretonnière $\mathrm{C}$, Jacqueline $\mathrm{C}$, Caillon $\mathrm{J}$, Guitton $\mathrm{C}$, Le Mabecque $\mathrm{V}$, Miégeville AF, et al. Efficacy of doripenem in the treatment of Pseudomonas aeruginosa experimental pneumonia versus imipenem and meropenem. J Antimicrob Chemother 2010;65:2423-7.

6. Walsh F. Doripenem: A new carbapenem antibiotic a review of comparative antimicrobial and bactericidal activities. Ther Clin Risk Manag 2007;3:789-94.

7. Collee JG, Fraser AG, Marmion BP, Simmons A. Tests for the identification of bacteria. In: Collee JG, Miles RS, Watt B, editors. Mackey and McCartney Practical Medical Microbiology. 14 $4^{\text {th }}$ ed. New Delhi: Elsevier; 2006. p. 131-49.

8. Clinical and Laboratory Standards Institute. Performance Standards for Antimicrobial Susceptibility Testing. Document; Twenty-Fifth International Supplement M100-S26. Wayne, PA: CLSI; 2016.

9. European Committee on Antimicrobial Susceptibility Testing (EUCAST). Breakpoint tables for interpretation of MICs and zone diameters. Ver. 6.0, 2016. EUCAST; 2016.

10. Magiorakos AP, Srinivasan A, Carey RB, Carmeli Y, Falagas ME, Giske CG, et al. Multidrug-resistant, extensively drug-resistant and pandrug-resistant bacteria: An international expert proposal for interim standard definitions for acquired resistance. Clin Microbiol Infect 2012;18:268-81.

11. Bretonnière C, Maitte A, Caillon J, Potel G, Boutoille D, Jacqueline C, et al. MIC score, a new tool to compare bacterial susceptibility to antibiotics application to the comparison of susceptibility to different penems of clinical strains of Pseudomonas aeruginosa. J Antibiot (Tokyo) 2016;69:806-810.

12. Kakeya H, Yamada K, Nakaie K, Takizawa E, Okada Y, Fujita A, et al. A comparison of susceptibility of Pseudomonas aeruginosa clinical isolates to carbapenem antibiotics in our hospital. Jpn J Antibiot 2014;67:241-8.

13. Goyal K, Gautam V, Ray P. Doripenem vs. meropenem against Pseudomonas and Acinetobacter. Indian J Med Microbiol 2012;30:350-1.

14. Hagihara M, Kuti JL, Nicolau DP. Predicting doripenem susceptibility based on meropenem and imipenem interpretation for Pseudomonas aeruginosa. Diagn Microbiol Infect Dis 2012;72:258-62.

15. Hojabri Z, Ahangarzadeh Rezaee M, Nahaei MR, Soroush MH, Ghojazadeh M, Pirzadeh T, et al. Comparison of in vitro activity of doripenem versus old carbapenems against Pseudomonas aeruginos clinical isolates from both CF and burn patients. Adv Pharm Bull 2013;3:121-5.

16. Li Y, Lv Y, Xue F, Zheng B, Liu J, Zhang J. Antimicrobial resistance surveillance of doripenem in China. J Antibiot (Tokyo) 2015;68:496-500.

17. Pillar CM, Torres MK, Brown NP, Shah D, Sahm DF. In vitro activity of doripenem, a carbapenem for the treatment of challenging infections caused by gram-negative bacteria, against recent clinical isolates from the United States. Antimicrob Agents Chemother 2008;52:4388-99.

18. Pragasam AK, Raghanivedha M, Anandan S, Veeraraghavan B. Characterization of Pseudomonas aeruginosa with discrepant carbapenem susceptibility profile. Ann Clin Microbiol Antimicrob 2016;15:12. 